\title{
Ion channel inhibition against COVID-19: A novel target for clinical investigation
}

\author{
Eliano P. Navarese ${ }^{1,2}$, Rita L. Musci ${ }^{3}$, Lara Frediani ${ }^{4}$, Paul A. Gurbel ${ }^{5}$, Jacek Kubica ${ }^{1}$ \\ ${ }^{1}$ Department of Cardiology, Collegium Medicum, Nicolaus Copernicus University, Bydgoszcz, Poland \\ and SIRIO MEDICINE Research Network \\ ${ }^{2}$ Faculty of Medicine University of Alberta, Edmonton, Canada \\ ${ }^{3}$ Department of Cardiology, Azienda Ospedaliera Bonomo, Andria, Italy \\ ${ }^{4}$ Department of Cardiology, Azienda Usl Toscana Nord-Ovest Cardiologia \\ UTIC-Ospedali Riuniti di Livorno, Italy \\ ${ }^{5}$ Sinai Center for Cardiovascular Research, Sinai Hospital of Baltimore, \\ LifeBridgehealth, Baltimore, MD, USA
}

\section{Introduction}

Infection with severe acute respiratory syndrome coronavirus 2 (SARS-CoV-2), also known as coronavirus disease (COVID-19), has been classified by the World Health Organization (WHO) as an ongoing pandemic. Owing to the global emergency, there is an unmet need to identify effective and scalable therapeutic options to attenuate the early stages of virus infection.

SARS-CoV-2 has different stages of the infection cycle. The first phase is characterized by infection and replication of the virus within the host cells. The last phase occurs with cytokine storm leading to cellular apoptosis [1].

We posit the rationale for ion channel inhibition as a novel therapeutic target to counteract SARS-CoV-2 infection and replication. Within this framework, we discuss the potential clinical role of the ion channel inhibitors amiodarone and verapamil against COVID-19 that deserves clinical investigations.

\section{Mechanisms of SARS-CoV-2 entrance into the host cell}

SARS-CoV-2 belongs to the family of Coronaviridae, which also includes severe acute respiratory syndrome coronavirus (SARS-CoV) and middle east respiratory syndrome coronavirus
(MERS-CoV). SARS-CoV-2 is highly contagious and is transmitted primarily via respiratory droplets.

A critical step in the life cycle of SARS-CoV-2 cell entry is the binding of viral spike protein (S protein) subunit S1 to angiotensin converting enzyme 2 (ACE2) receptors that are expressed mainly in human alveolar cells, and protein $\mathrm{S}$ priming by host proteases. This process leads to fragmentation in the S1 and S2 subunit interface and catalyzes a membrane fusion reaction [2]. The S2 subunit in turn promotes the fusion of the viral envelope with the host cell membrane [2]. Transmembrane protease, serine 2 (TMPRSS2) cleaves and activates the viral spike glycoproteins, which in turn facilitates virus-cell membrane fusion.

The conformational modification of viral envelope with $\mathrm{S}$ protein exposure and fusion with host cell membrane constitute the initial phase of cell entry.

The spike protein therefore plays a dual role in promoting virus entry by mediating receptor binding and then membrane fusion. This phase can be termed "early-entry" of the virus infection.

In the absence of exogenous or membranebound proteases that enable entry within the plasma membrane surface, coronaviruses can be internalized via clathrin- and non-clathrin-mediated endocytosis [3], where the $S$ protein is cleaved by cathepsin L, which promotes fusion of viral mate-

Address for correspondence: Prof. Eliano P. Navarese, MD, PhD, FESC, FACC, Interventional Cardiology and Cardiovascular Medicine Research, Department of Cardiology and Internal Medicine, Nicolaus Copernicus University, ul. Skodowskiej-Curie 9, 85-094 Bydgoszcz, Poland, tel: +48 52585 4023, fax: +48 52585 4024, e-mail: elianonavarese@gmail.com 


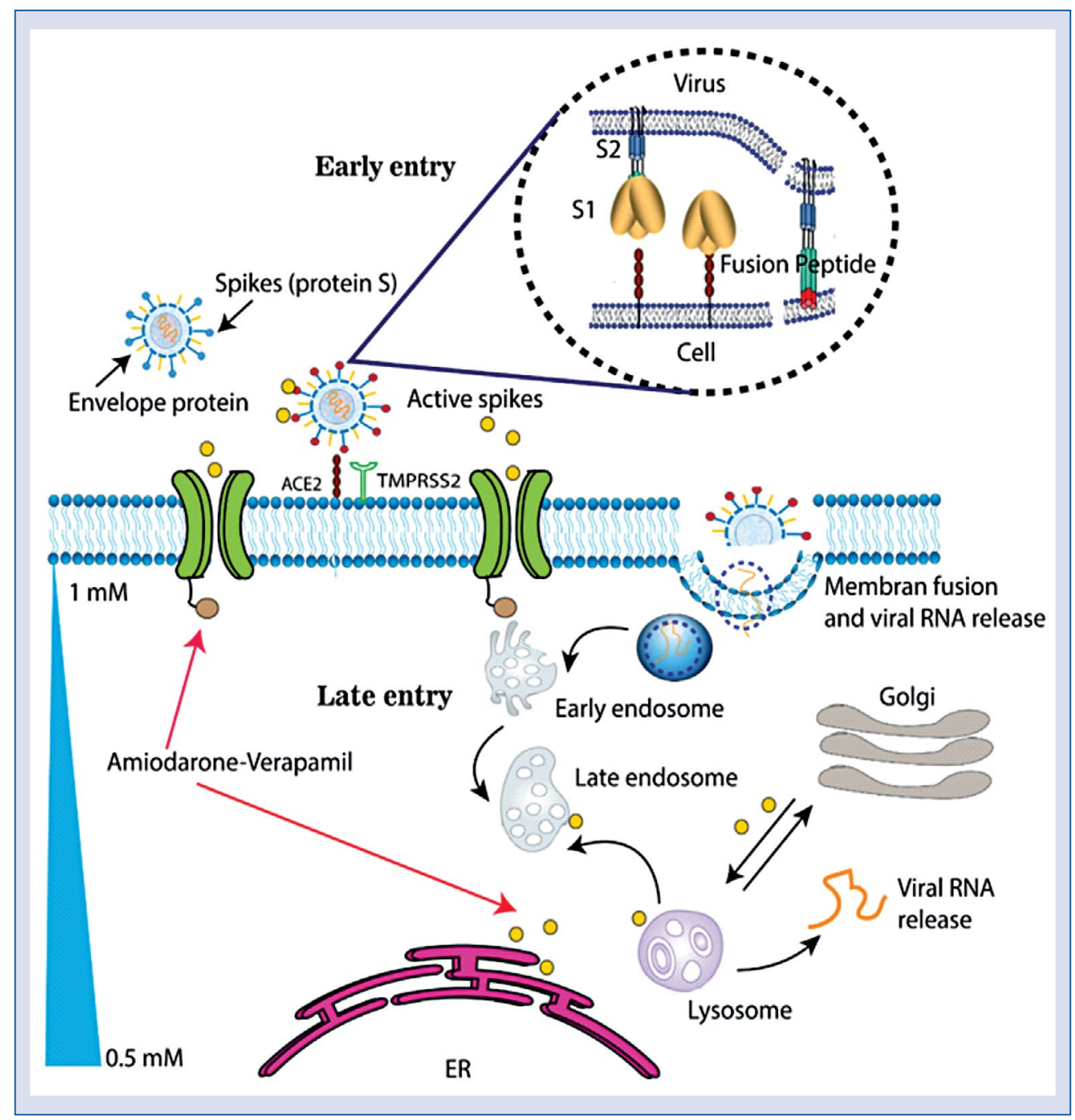

Figure 1. COVID-19 mechanisms of entry. COVID-19 entrance into the host cell takes place in two phases: "early entry" and "late entry". In the "early entry" the viral S protein-subunit S1 binds the angiotensin converting enzyme 2-receptors (ACE2-receptors) on human cells and transmembrane protease-serine 2 (TMPRSS2) facilitates virus-cell membrane fusion. The $\mathrm{Ca}^{2+}$ ions promote viral membrane fusion and $\mathrm{S}$ protein conformational changes which allow the fusion peptide insertion into the lipid bilayer. In the "late entry" COVID-19 is endocytosed and $\mathrm{Ca}^{2+}$ ions have an important role in endocytic vesicles maturation. This process ends with the release of the viral genome into cytoplasm and the subsequent replication of the virus. Amiodarone and verapamil block $\mathrm{Ca}^{2+}$ cell membrane and endosomal/ /lysosomal channels interfering with the life-cycle of coronavirus; RNA — ribonucleic acid; S protein — spike protein; S1-S2 - subunit 1 and subunit 2 of S protein; ER - endosplamic reticulum.

rial with endosomal membranes ("late entry"). The final common step is the release of the viral genome into cytoplasm and the subsequent replication of the virus.

These entry mechanisms are shared by the new SARS-CoV-2 with the other members of coronavirus family. Fusion peptides of SARS-CoV and SARS-CoV-2 have been found highly comparable sharing more than $90 \%$ homologies in their biological sequences.
Ion channels are multi-subunit, pore-forming membrane proteins that mediate the rapid and selective passage of ions across all cell membranes.

The underlying hypothesis is that SARS-CoV-2 virion entry, host-membrane fusion and the ensuing virus replication in the host cells are governed by ion currents. Within this framework, $\mathrm{Ca}^{2+}$ ions are necessary to promote fusion peptide insertion into the lipid bilayer and the endocytosis pathway (Fig. 1). Pharmacological agents that 
target ion channels may therefore modulate virus life cycles.

\section{Ion channel regulation of virus life cycle}

\section{Early cell entry}

A role of $\mathrm{Ca}^{2+}$ in viral membrane entry and fusion has been reported. Proteolytic cleavage of the $S$ protein exposes its fusion peptide and initiates membrane fusion. It has been shown that $\mathrm{Ca}^{2+}$ plays an active role in stimulating the fusogenic activity of the SARS-CoV fusion peptide via a $\mathrm{Ca}^{2+}$ binding pocket [4]. Other experiments have tested the ability of virus pseudo-particles to mediate infection of host cells without and with $\mathrm{Ca}^{2+}$ and have shown that intracellular $\mathrm{Ca}^{2+}$ enhances MERS-CoV infection by approximately two-fold [5].

Infectivity assays with pseudoparticles expressing SARS-CoV and MERS-CoV S protein demonstrated that SARS-CoV and MERS-CoV entry into host cells was reduced when intracellular $\mathrm{Ca}^{2+}$ was chelated [6]. SARS-CoV and MERS-CoV further promote virus entry triggering a process termed membrane ordering that induces a rearrangement of membrane lipid bilayer, enhanced in the presence of $\mathrm{Ca}^{2+}[7,8]$.

Together, these observations lend support to the hypothesis that $\mathrm{Ca}^{2+}$ plays an active role in inducing viral membrane fusion. The spike proteins of the SARS-CoV-2 are more robust and resistant than previous members of the coronavirus family.

$\mathrm{Ca}^{2+}$ ions interacting with the fusion peptide can induce spatial changes in $\mathrm{S}$ protein altering the fusion peptide's structure, and interaction with the cell membrane, promoting infection in MERS-CoV, SARS-CoV and SARS-CoV-2 [7].

\section{Endocytosis (endosome-maturation- -late entry)}

Endoplasmic reticulum (ER), reticulum-Golgi apparatus and lysosomes are vital components of the host cell machinery used by the SARS-CoV-2 [9]. Spike, envelope and membrane proteins enter the $\mathrm{ER}$, and the nucleocapsid protein is combined with the $(+)$ strand genomic ribonucleic acid (RNA) to become a nucleoprotein complex. They merge into a complete virus particle in the host endoplasmic ER-Golgi apparatus compartment. $\mathrm{Ca}^{2+}$ has been found to exert major modulatory roles on ER and lysosomes [10]. While extracellular $\mathrm{Ca}^{2+}$ is high, levels drop rapidly in the lumen of newly formed endocytic vesicles, due to the action of efflux pumps [11]. During later stages of endosome maturation, lysosome-late endosome fusion implies the release of $\mathrm{Ca}^{2+}$ from the lumen of endocytic organelles, which is used to mediate membrane fusion events at several stages on the endocytic pathway [12]. Such trafficking pathways can therefore lead to the availability of $\mathrm{Ca}^{2+}$ during the early entry of the coronavirus and afterwards during the late phase of mature endocytic vesicle formation.

\section{Clinical utility of amiodarone and verapamil in patients with COVID-19}

Amiodarone is a benzofuran derivative, an anti-arrhythmic drug commonly used in a variety of settings and is approved for the treatment of ventricular arrhythmias and atrial fibrillation. As a class III antiarrhythmic agent, amiodarone is a non-selective ion channel inhibitor that blocks $\mathrm{Ca}^{2+}, \mathrm{Na}^{+}$and $\mathrm{K}^{+}$voltage-gated channels. Amiodarone binds to and then blocks $\mathrm{Ca}^{2}$-channels predominantly during the inactive or resting state associated with the suppression of $\mathrm{Ca}^{2}$-dependant action potentials. Verapamil is a prototypical phenylalkylamine, which exerts antihypertensive, antiarrhythmic and antianginal effects. Verapamil selectively inhibits intracellular transmembrane $\mathrm{Ca}^{2+}$ flow through L-type voltage-gated $\mathrm{Ca}^{2+}$ channels.

Previous studies have shown the effects of amiodarone on endosomal transport in SARS-CoV-infected cells by blocking $\mathrm{Ca}^{2+}$ channels [13]. Other in vitro data showed that amiodarone and verapamil, at concentrations routinely reached in human serum can, when employed clinically, act as a host cell-targeting agent that block filovirus entry [14]. These results confirmed that $\mathrm{Ca}^{2+}$ channel activity is required during virion entry processes [14].

Another study demonstrated that amiodarone interferes with the fusion of the Ebola viral envelope with the endosomal membrane at concentrations close to those found in patients treated for arrhythmias; an additive inhibitory effect of amiodarone and its pharmacologically active metabolite monodesethyl amiodarone on entry into target cells has been noted [15].

\section{Clinical perspectives}

Modulation of host cell ion channel activity by viral proteins is being increasingly identified as an important virus-host interaction. With this background, an ongoing randomized clinical trial (clinicaltrials.gov ID: NCT04351763), will investigate the role of amiodarone and verapamil to inhibit 
ion channels in hospitalized patients with proven COVID-19. There is an urgent need to identify stable, effective and scalable therapeutic options against initial stages of virus infection and replication. Ion channel inhibition with the cardiovascular agents amiodarone and verapamil might reduce the severity of disease and the transmission potential of COVID-19.

\section{Conflict of interest: None declared}

\section{References}

1. Siddiqi HK, Mehra MR. COVID-19 illness in native and immunosuppressed states: A clinical-therapeutic staging proposal. J Heart Lung Transplant. 2020; 39(5): 405-407, doi: 10.1016/j. healun.2020.03.012, indexed in Pubmed: 32362390.

2. Hoffmann M, Kleine-Weber H, Schroeder S, et al. SARS-CoV-2 cell entry depends on ACE2 and TMPRSS2 and is blocked by a clinically proven protease inhibitor. Cell. 2020; 181(2): 271-280. e8, doi: 10.1016/j.cell.2020.02.052.

3. Inoue $\mathrm{Y}$, Tanaka $\mathrm{N}$, Tanaka $\mathrm{Y}$, et al. Clathrin-dependent entry of severe acute respiratory syndrome coronavirus into target cells expressing ACE2 with the cytoplasmic tail deleted. J Virol. 2007; 81(16): 8722-8729, doi: 10.1128/JVI.00253-07, indexed in Pubmed: 17522231

4. Lai AL, Millet JK, Daniel S, et al. The SARS-CoV fusion peptide forms an extended bipartite fusion platform that perturbs membrane order in a calcium-dependent manner. J Mol Biol. 2017; 429(24): 3875-3892, doi: 10.1016/j.jmb.2017.10.017, indexed in Pubmed: 29056462.

5. Straus MR, Tang T, Lai AL, et al. Ca ions promote fusion of middle east respiratory syndrome coronavirus with host cells and increase infectivity. J Virol. 2020; 94(13), doi: 10.1128/JVI.00426-20, indexed in Pubmed: 32295925.

6. Qian Z, Dominguez SR, Holmes KV. Role of the spike glycoprotein of human Middle East respiratory syndrome coronavirus
(MERS-CoV) in virus entry and syncytia formation. PLoS One. 2013; 8(10): e76469, doi: 10.1371/journal.pone.0076469, indexed in Pubmed: 24098509.

7. Tang T, Bidon M, Jaimes JA, et al. Coronavirus membrane fusion mechanism offers a potential target for antiviral development. Antiviral Res. 2020; 178: 104792, doi: 10.1016/j.antiviral.2020.104792, indexed in Pubmed: 32272173.

8. Basso LGM, Vicente EF, Crusca E, et al. SARS-CoV fusion peptides induce membrane surface ordering and curvature. Sci Rep. 2016; 6: 37131, doi: 10.1038/srep37131, indexed in Pubmed: 27892522.

9. de Haan CAM, Rottier PJM. Molecular interactions in the assembly of coronaviruses. Adv Virus Res. 2005; 64: 165-230, doi: 10.1016/S0065-3527(05)64006-7, indexed in Pubmed: 16139595.

10. Raffaello A, Mammucari C, Gherardi G, et al. Calcium at the center of cell signaling: interplay between endoplasmic reticulum, mitochondria, and lysosomes. Trends Biochem Sci. 2016; 41(12): 1035-1049, doi: 10.1016/j.tibs.2016.09.001, indexed in Pubmed: 27692849.

11. Huotari J, Helenius A. Endosome maturation. EMBO J. 2011; 30(17): 3481-3500, doi: 10.1038/emboj.2011.286, indexed in Pubmed: 21878991.

12. Pryor PR, Mullock BM, Bright NA, et al. The role of intraorganellar $\mathrm{Ca}(2+)$ in late endosome-lysosome heterotypic fusion and in the reformation of lysosomes from hybrid organelles. J Cell Biol. 2000; 149(5): 1053-1062, doi: 10.1083/jcb.149.5.1053, indexed in Pubmed: 10831609.

13. Stadler K, Ha HR, Ciminale V, et al. Amiodarone alters late endosomes and inhibits SARS coronavirus infection at a post-endosomal level. Am J Respir Cell Mol Biol. 2008; 39(2): 142-149, doi: 10.1165/rcmb.2007-02170C, indexed in Pubmed: 18314540.

14. Gehring G, Rohrmann K, Atenchong N, et al. The clinically approved drugs amiodarone, dronedarone and verapamil inhibit filovirus cell entry. J Antimicrob Chemother. 2014; 69(8): 2123-2131, doi: 10.1093/jac/dku091, indexed in Pubmed: 24710028.

15. Salata C, Baritussio A, Munegato D, et al. Amiodarone and metabolite MDEA inhibit Ebola virus infection by interfering with the viral entry process. Pathog Dis. 2015; 73(5), doi: 10.1093/ femspd/ftv032, indexed in Pubmed: 25933611. 